\title{
El mercado institucional de centros de educación pública para productos orgánicos en Costa Rica
}

\section{The institutional market of public education centers for organic products in Costa Rica}

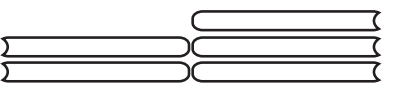

Carlos Soto Padilla ${ }^{1}$

Recibido: octubre 2016 Aprobado: mayo 2017

\section{Resumen}

La agricultura orgánica en Costa Rica no ha logrado perfilarse como una actividad productiva viable y rentable para la pequeña producción agropecuaria dentro de una estrategia de desarrollo rural, a falta de voluntad política de los gobiernos y la inexistencia de mercados nacionales asequibles, que consideren las limitaciones organizativas, empresariales y comerciales que caracterizan a las organizaciones de pequeños productores orgánicos. Este postulado motivó la investigación sobre mercados alternativos existentes para estos productos en el ámbito nacional, y la realización de un piloto comercial a partir de sus hallazgos, entre la Asociación de Productores Orgánicos de la Zona Norte de Cartago (APROZONOC) y el Liceo Experimental Bilingüe José Figueres Ferrer, en la perspectiva de una activa y efectiva participación de ambos actores, fundamentado en el enfoque de desarrollo denominado "desde abajo y desde adentro", contrapuesto a los enfoques de desarrollo "desde afuera y desde arriba", históricamente implementados en América Latina, y fracasados en su objetivo de mejorar la condición de vida de las poblaciones de los espacios rurales, al ser elaborados y ejecutados a partir del Estado o del mercado, sin mediar la consulta ni la participación de las mayorías en su gestión y realización.

Palabras clave: Pequeños productores orgánicos, desarrollo rural desde abajo y desde adentro, producción orgánica, mercados institucionales para productos orgánicos.

\begin{abstract}
In Costa Rica, organic agriculture has not been able to establish itself as a viable and profitable activity for small farmers within a rural development strategy, lacking political will from the
\end{abstract}

1 Maestría en Desarrollo Rural, UNA, Heredia, Costa Rica. sotopadilla57@gmail.com 
government and the absence of accessible national markets, that consider organizational, corporate and commercial limitations associated with small farmer organizations.

This postulate motivated the investigation about existing alternative markets for these products within the national scope, and the fulfillment of a commercial pilot with its discoveries, between APROZONOC and the Jose Figueres Ferrer Experimental and Bilingual high school, in the perspective of an active and effective participation from both parties, based on a coming from the bottom and from within development, against a foreign and "coming from the top", which have generally failed as the development models implemented in Latin America, in its objective to improve the lifestyle of the population from rural areas, implemented parting from either the nation state or the market, without taking into account the majority's opinion in its management and execution.

Keywords: Small organic farmers, coming from the bottom and within rural development, organic farming, institutional markets for organic products.

\section{Introducción}

Según Armesto (2007), existe una convergencia entre el desarrollo rural y la agricultura orgánica, en cuyo caso el término sostenibilidad es el punto de encuentro, pues en su definición aparecen las premisas de crecimiento económico, la mejora de las condiciones sociales, el respeto, y la conservación y mejora de los valores naturales; todo lo cual debe basarse en el uso de los recursos endógenos, que debe surgir desde un enfoque en el que la población local sea la protagonista e inductora de los cambios, y la máxima receptora de los beneficios que reporten estos procesos.

En este sentido, De la Cruz y Ramírez (2011) señalan que la agricultura orgánica es una estrategia de desarrollo, que trata de cambiar limitaciones encontradas en la producción convencional; es, más que una tecnología de producción, una práctica fundamentada en el mejor manejo del suelo, el fomento del uso de insumos locales, y en un mayor valor agregado, no solo económico, sino también en la conformación de nichos de mercado.

En el caso de Costa Rica, el crecimiento de la producción orgánica se ha visto limitada por la falta de voluntad política de los gobiernos, y en nuestro criterio, por la ausencia de mercados nacionales. 
Aceptando como válidas estas aseveraciones, cabe cuestionarse y revisar acerca de la existencia, accesibilidad e importancia de mercados para la comercialización de productos orgánicos, más allá de las ferias de agricultores, supermercados y otras manifestaciones comerciales de menor impacto en el ámbito nacional, en términos de las posibilidades y oportunidades que puedan presentar, para la generación de ingresos que permitan la reproducción de los sistemas productivos, el crecimiento de la producción orgánica y mejorar la calidad de vida de los grupos pequeños productores y sus familias.

En esta dirección, identificamos un nicho comercial como el pretendido en los mercados institucionales, particularmente en el del Programa de Alimentación y Nutrición del Escolar y del Adolescente (PANEA) del Ministerio de Educación Pública (MEP), no solo por estar favorecido por una ley de la República, sino también por su forma de operar, su gran demanda de alimentos y el presupuesto asignado, con montos que aumentaron, según Oviedo (2010), de los $\$ 6$ mil millones (US $\$ 11,857.708$ ) en el 2006 a $\$ 10$ mil millones (US\$18,050.541) en el 2009; y de $\$ 364,291.840$ (US\$708.739) en el 2014, solo para la provincia de Cartago, área de influencia del estudio realizado.

A partir de la problemática expuesta y la posibilidad de solución encontrada, se desarrolló una experiencia de piloto comercial entre la Asociación de Productores Orgánicos de la Zona Norte de Cartago (APROZONOC) y el Liceo Experimental Bilingüe José Figueres Ferrer, para valorar los beneficios de este mercado para los grupos productores orgánicos, validar el proceso comercial a desarrollar en la perspectiva de una metodología para su réplica en otras regiones del país, aportar a la expansión de la producción orgánica en el ámbito nacional y estimar su potencial dentro de una estrategia de desarrollo rural.

\section{La agricultura orgánica en el contexto nacional}

Hasta el año 2000, la producción orgánica en Costa Rica era poco conocida por la generalidad de la población. Esta limitada demanda comercial ha ido creciendo progresivamente hasta absorber la escasa oferta nacional, que marca un desabastecimiento que se mantiene hasta el presente 2016.

Según Amador (2005), el crecimiento de la agricultura orgánica costarricense ha sido espontáneo y desordenado en función del mercado externo, e inducido hacia el mercado interno por la preocupación de público consumidor interesado en la alimentación sana, y de otros actores que han observado, en esta actividad, una alternativa de desarrollo ante los desatinos de la revolución verde. 
Como evidencia del lento crecimiento de la producción orgánica en Costa Rica, el MAG (2014) reportó para el año 2000 una superficie de 8600 ha, que mantuvo un crecimiento sostenido hasta un máximo de 11.115 has en el 2010, año a partir del cual comenzó a decrecer paulatinamente hasta las 7832 ha en el 2014; es, a su vez, la estimación de la superficie orgánica dentro del total agrícola del país de $0,4 \%$ y un número de 3000 sujetos productores dedicados a la actividad (FiBL, 2015).

Según el MAG (2013), el 69\% de la producción nacional de alimentos orgánicos se destina a la exportación y el 31\% al mercado nacional, sin mediar estrategia alguna para su comercialización.

Considerado este $31 \%$ como el $100 \%$ del producto orgánico que se coloca en el mercado local, encontramos en las ferias del agricultor con un $35 \%$ y en los entes intermediarios con un 20\%, los principales canales de comercialización de la actividad, mientas el restante $45 \%$ de la oferta nacional se coloca en espacios comerciales de menor importancia como restaurantes $15 \%$, supermercados $10 \%$, puntos de venta diversos $10 \%$ y el mercado convencional con un $10 \%$ (MAG, 2013).

En la generalidad de estos canales de comercialización, se reportó una mayor venta de productos orgánicos en transición ${ }^{2}$ y como producto convencional, situación que puede relacionarse con el alto costo de la certificación ${ }^{3}$, que para el caso de un sujeto productor puede alcanzar, según el IICA (2010), los US\$800.

En términos de tamaño, Pérez (2014, julio 22) reseñó que el Centro de Inteligencia de Mercados Sostenibles del INCAE estimó que el mercado orgánico en Costa Rica, para el año 2014, rondaba los US\$500.000 anuales con un crecimiento del 10\%; superior a lo establecido por Granados (2005) de US $\$ 300.000$ anuales durante el periodo 2000 al 2005, con igual porcentaje de crecimiento anual (5 - 10\%).

Particularmente sobre el tema de mercados nacionales, el artículo 48 del reglamento a la Ley para el Fomento de la Producción Agropecuaria Orgánica en Costa Rica, establece que el Departamento de Fomento para la Producción Agropecuaria Orgánica dará a conocer los beneficios del consumo de productos orgánicos a las instituciones centralizadas y descentralizadas del Estado y a sus proveedores, entre ellos el PANEA del MEP, para lo cual coordinará

2 Tiempo que debe transcurrir en el tránsito de un sistema de producción convencional (con la utilización de agroquímicos) a un sistema orgánico, de acuerdo con un plan de transformación previamente establecido.

3 Documento que da fe de que el producto que ampara ha cumplido en todas sus etapas con los principios, las normativas y requisitos vigentes para la producción orgánica. 
con los jerarquías respectivas, la decisión de compra de bienes y servicios provenientes de este sector.

Asimismo, este departamento servirá de enlace entre los productores y las instituciones interesadas, para que programen las ventas y negocien un precio diferenciado para dichos productos; acciones que al 2016 no han sido ejecutadas, por la inexistencia de este departamento, cuya creación estaba decretada para el 2009.

De esta manera, el letargo que presenta la producción orgánica y su mercado en el espacio nacional ha coartado la pretensión de los diferentes gobiernos en Costa Rica, a partir de los años 90, con el modelo de desarrollo de la Nueva Ruralidad, de incluir, sin lograrlo, la actividad orgánica dentro de los planes de desarrollo rural.

\section{El mercado institucional en Costa Rica}

Según el Consejo Nacional de la Producción (CNP) (2012), el mercado institucional en Costa Rica tuvo su origen en 1977 como el Programa de Abastecimiento Institucional (PAI) del CNP, y presenta, desde el inicio, problemas de orden administrativo y presupuestario -que se mantienen hasta hoy- en la atención de un mercado estimado en más de 73 mil millones de colones (US\$137 millones), de los cuales el CNP retiene un $21 \%$ para su funcionamiento.

\section{El programa de alimentación escolar en Costa Rica}

El PANEA es un programa del MEP creado con el objetivo de brindar una alimentación complementaria y la promoción de hábitos alimentarios saludables en la población estudiantil, además de ofrecer subsidios para la compra de alimentos, la contratación de personal para los comedores estudiantiles, su equipamiento, las mejoras a sus infraestructuras, el establecimiento de proyectos productivos, el apoyo con material técnico y didáctico, la capacitación para el desarrollo de huertas estudiantiles y la educación para la salud.

Por nivel educativo, los comedores escolares dentro del PANEA atienden principalmente a la población de primaria (79\%); con un total de individuos beneficiarios de los comedores escolares de 470.657 estudiantes, un 53\% pertenecientes a zonas rurales y el otro $47 \%$ a zonas urbanas.

En cuanto al nivel de pobreza de la población beneficiaria, el 36\% corresponde a pobres, un $11 \%$ se encuentra en extrema pobreza, el $26 \%$ no satisface sus necesidades básicas y $64 \%$ se cataloga como no pobres. 
Por nivel educativo, el $4 \%$ corresponde a preparatoria, un $79 \%$ a primaria y el $16 \%$ a secundaria (Oviedo, 2010).

\section{Localización geográfica y clima de la zona de estudio}

El piloto comercial se realizó en la provincia de Cartago, Costa Rica, cuya superficie es de $4547 \mathrm{~km}^{2}$ (11,6\% del territorio nacional), con una precipitación anual de 1500 - $2000 \mathrm{~mm}$ y temperaturas promedio anuales de $19,2^{\circ} \mathrm{C}$, como resultado de una altitud intermedia de $1435 \mathrm{msnm}$ (figura 1).

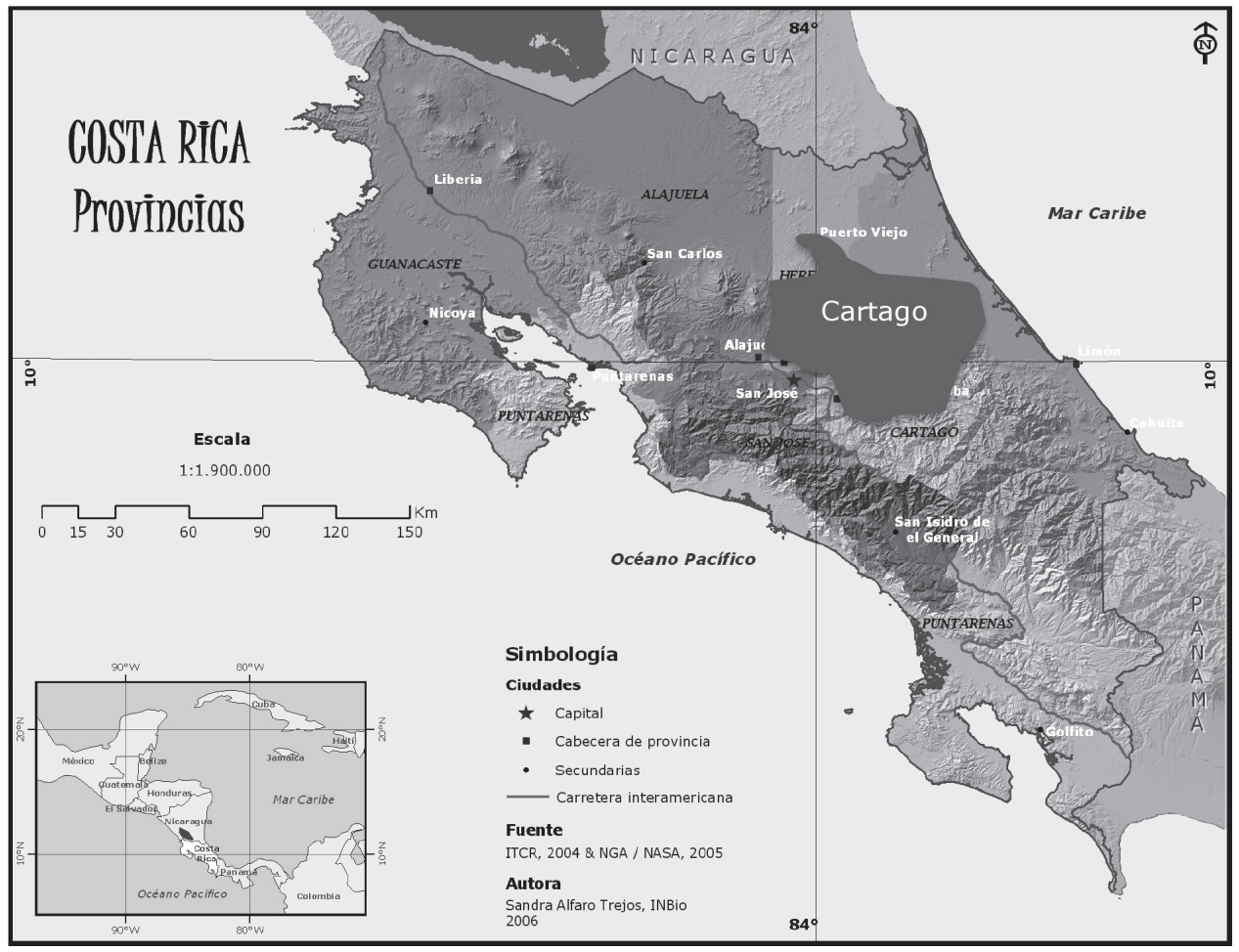

Figura 1. Localización geográfica de la provincia de Cartago en el territorio de Costa Rica. Aspectos socioeconómicos.

La provincia de Cartago es la zona más importante del país en el cultivo de hortalizas y otros cultivos de zonas templadas.

Con las medidas económicas adoptadas por los gobiernos de los últimos 30 años, la mayoría de los sujetos productores han tenido grandes pérdidas en sus explotaciones, y presentan problemas de bajo desarrollo tecnológico, altos costos de producción, bajo nivel organizativo de los grupos productores, sistemas de agrocadenas poco desarrollados, asistencia técnica insuficiente, baja rentabilidad de la producción por fluctuaciones en los precios de los productos en el 
mercado, altos costos de producción, plagas, carencia de proyectos de riego y el poco desarrollo y diversificación de la agroindustria. Adicionalmente, las organizaciones de grupos productores enfrentan problemas de reducida capacidad gerencial y administrativa, que no permite contar con una autogestión eficiente, para reducir los márgenes de intermediación a favor del ente productor.

Asimismo, estas organizaciones disponen de poco capital de trabajo y dificultades para el acceso al crédito formal, además de presentar una débil cartera de proyectos productivos, que les permita generar ingresos y un mejor desarrollo para sus comunidades.

\section{Los sujetos participantes de la investigación}

Los sujetos participantes del presente estudio son los miembros de la APROZONOC, una organización constituida en 1997, conformada al 2015 por 22 personas socias; y el Liceo Bilingüe Experimental José Figueres Ferrer, una institución pública fundada en 1995, con una población estudiantil al 2014, compuesta de 710 estudiantes, 130 que utilizaba el servicio de comedor y 100 con beca alimentaria del PANEA.

\section{Pasos metodológicos del piloto comercial}

Paso 1. Selección de los sujetos participantes para el piloto comercial

a. Establecimiento de criterios para la selección de la organización de sujetos productores.

b. Definición de criterios para la selección del centro educativo.

Paso 2. Elaboración, planteamiento y negociación de la propuesta del piloto comercial con los sujetos participantes seleccionados para su aprobación, ajuste o rechazo ${ }^{4}$

Paso 3. Líneas de acción

a. Determinación de la demanda del centro educativo.

b. Inventario de la oferta de la organización de productores.

c. Cualificación de la capacidad organizativa, técnica y material de la organización de productores para asumir el piloto comercial.

d. Definición de los términos de negociación para la implementación del piloto comercial.

4 Eventualmente y a discreción de los sujetos participantes, la propuesta de piloto puede ser presentada a otros actores claves de los sectores público, privado, académico, de la sociedad civil o la cooperación internacional, identificados como potenciales alianzas o sinergias, para valorar su interés y pertinencia de participación en este. 
Paso 4. Operación del proceso de comercialización

a. Recibo de la APROZONOC del pedido de productos demandados por el centro educativo.

b. Consulta y distribución del pedido de productos entre los miembros de la organización de productores, según existencias y capacidad productiva.

c. Preparación de los productos para su embalaje y transporte al centro educativo.

a. Entrega de los productos en el centro educativo, revisión y aprobación por responsables del comedor.

b. Facturación y cancelación del pedido de productos.

Paso 5. Evaluación del proceso comercial desarrollado

a. Elaboración de instrumentos de evaluación.

b. Aplicación del instrumento de evaluación al centro educativo.

c. Aplicación del instrumento de evaluación a la organización de productores.

d. Tabulación de la información, valoración de resultados y retroalimentación del piloto comercial.

\section{Principales hallazgos de la investigación}

El piloto comercial desarrollado encontró sustento operativo y legal en la Ley de Fomento para la Producción Orgánica y en el reglamento del PANEA del MEP.

Esta experiencia comercial, inédita en Costa Rica, generó una metodología reproducible con las consideraciones del caso, en otras regiones del país.

Sin embargo, su réplica, desde el punto de vista legal, es impracticable, dada la inexistencia del Departamento de Fomento a la Producción Agropecuaria Orgánica, responsable de ejecutar los artículos 47 y 48 del reglamento de la Ley para el Fomento de la Actividad Orgánica.

Los efectos de la ausencia de este departamento y, por lo tanto, de la ejecución de las acciones que le fueron asignadas, quedaron manifiestas en el desconocimiento de la actividad orgánica en el país por parte del centro educativo y del decreto para el desarrollo de los mercados institucionales para estos productos, desconocido tanto por parte de la institución educativa como de la APROZONOC. Asimismo, estos últimos desconocían la existencia del 
PANEA, su reglamento, y los trámites para concursar como instancias proveedoras de alimentos en los centros educativos.

Observaciones hechas al artículo 48, las emplazamos por una parte, en los vacíos observados en la falta de atención a la capacidad organizativa, empresarial y comercial de los grupos pequeños productores para vincularse a los mercados institucionales y, por otra parte, en la particularización exclusiva de los términos de negociación en el tema de precios y su diferenciación, cuando lo experimentado entre la APROZONOC y el centro educativo advirtió que la negociación además de los precios, considera las características de oferta, demanda y las capacidades de los entes productores para llevar adelante el proceso comercial.

Asimismo, establece un sobreprecio, sin consideraciones previas de costos de producción y comercialización, que podría incidir en una mayor justicia de precios para las partes.

En el tema productivo, la posibilidad de comercializar en un mercado a partir de su demanda y no desde la oferta, como ha sido la práctica histórica de los grupos pequeños productores, le permitió a la APROZONOC reducir sus costos de producción y comercialización, con una mayor optimización en la utilización de las formas, medios y recursos para la producción. Análogamente, esta condición permitió materializar las diferencias significativas en estos aspectos entre los socios de la APROZONOC y ratificó, así, la importancia de la planificación de los sistemas productivos en función del mercado.

Aun cuando la APROZONOC comprobó su capacidad de oferta y tecnología para atender la demanda del centro educativo, fue necesaria y estratégica la alianza con organizaciones amigas de productores de otras regiones para complementar la oferta; se demostró, así, la capacidad del piloto comercial para generar tejido social y la posibilidad de establecer una red de abastecimiento de productos entre regiones y se favoreció la participación de otra organización de productores.

Desde el punto de vista organizativo, los vacíos observados en la APROZONOC evidenciaron grandes limitaciones en las áreas comercial y empresarial para el desarrollo del piloto comercial, lo que al cabo del tiempo condicionaría su continuidad y sostenibilidad operativa.

Sin embargo y a pesar de los problemas organizativos presentados, la APROZONOC consiguió la creación de una comisión para la comercialización de los productos con el centro educativo, logrando, por vez primera en la 
historia de la organización, hacerlo de manera colectiva, y pudiendo negociar exitosamente su política de precios fijos para sus productos a través de todo el año, la colocación de productos en transición y la reducción de los costos de comercialización del piloto, lo que la bibliografía define como circuitos cortos de comercialización (CCC). (LEADER, julio, 2000).

A pesar de estos logros y como era previsible por la problemática organizativa presentada, la comisión de comercialización fue paulatinamente dejando de funcionar hasta su desaparición total, y quedó como responsabilidad del presidente de la asociación la continuación de proveer productos al centro educativo.

Siempre en el plano organizativo, fue importante el acercamiento de la junta directiva de la APROZONOC con el Banco Nacional de Costa Rica y el Instituto Tecnológico de Costa Rica, donde se dejó evidenciada la importancia del establecimiento de alianzas y sinergias y la conformación de redes entre sectores e instituciones y al interior de estos mismos, para los fines del piloto comercial.

En términos de la evaluación hecha de la experiencia comercial, la selección a priori de los sujetos participantes facilitó el balance positivo de los resultados que se obtuvieron.

Tanto el centro educativo como la APROZONOC valoraron positivamente la experiencia realizada con el piloto comercial, resaltando la asequibilidad del mercado académico.

Más allá de la venta de productos y los beneficios económicos para la APROZONOC, el piloto comercial proyectó valores agregados entre los que sobresalen:

- La posibilidad de establecer un sistema de garantía participativa ${ }^{5}$ con la participación de estudiantes.

- La proyección, reconocimiento y reivindicación de los grupos pequeños productores orgánicos como entes proveedores de alimentos sanos a la sociedad.

- La valoración del origen local de los alimentos, traducido en la preferencia por apoyar el esfuerzo de grupos productores de la región.

- La posibilidad para el centro educativo de complementar los contenidos curriculares con las visitas de estudiantes a las fincas.

$5 \quad$ Sistema de garantía desarrollado mediante una relación directa entre la persona o las personas productoras orgánicas y la persona o las personas consumidoras u otros actores interesados, quienes, garantizan el origen y la condición de los productos orgánicos destinados al mercado nacional. 


\section{Referencias}

Amador, M. (octubre, 2005). Mercado nacional de productos orgánicos. Revista electrónica Ambientico, (145), 10-11. Recuperado de http://www. ambientico.una.ac.cr/pdfs/ambientico/145.pdf

Armesto, X. (2007). El concepto de agricultura ecológica y su idoneidad para fomentar el desarrollo rural sostenible. Boletín electrónico de la Asociación de Geógrafos Españoles, 47, 155-172. Recuperado de http://www. dialnet.unirioja.es/descarga/articulo/2499261/1.pdf

Consejo Nacional de la Producción (CNP). (2012). Nuevo mercado institucional. Recuperado de http://www.cnp.go.cr/agronegocios/pai/Plan_Mercado_Institucional_PAI.pdf

De la Cruz, S., \& Ramírez, V. (febrero, 2011). Mercados orgánicos locales como una opción para los pequeños productores. Revista Electrónica Artículos y Ensayos de Sociología Rural, 10, 95-104. Recuperado de http://www.portal.chapingo.mx/sociologia/doc/rae/articulo\%20y\%20 ensayos_10.pdf

FiBL. (2015). The world of organic agriculture: Statistics \& emerging trends 2015. Recuperado de https://www.fibl.org/fileadmin/documents/ shop/1663-organic-world-2015.pdf

Granados, E. (2005). Actualidad de la agricultura orgánica en Costa Rica. Recuperado de http://www.ambientico.una.ac.cr/145.pdf

IICA. (2010). Sistemas de garantía para productos orgánicos en mercados locales y nacionales. Recuperado de http://www.iica.ac.cr/Esp/Programas/ Innovacion/Publicaciones_TeI/B1822e.pdf

LEADER. (julio, 2000). Comercialización de los productos locales: Circuitos cortos y circuitos largos. "Innovación en el medio rural". Cuaderno de la innovación (7) Recuperado de http://ec.europa.eu/agriculture/rur/ leader2/rural-es/biblio/circuits/circuits.pdf

Ministerio de Agricultura y Ganadería. (2014). Estadísticas País: Costa Rica. Recuperado de https://www.sfe.go.cr/perfiles/exportacion/arao/Estadisticas_2014.pdf

Ministerio de Agricultura y Ganadería [MAG]. (2013). Estudio sobre el entorno nacional de la agricultura orgánica en Costa Rica. Recuperado de 
http://www.mag.go.cr/biblioteca_virtual/bibliotecavirtual/prog-nacagric-org-entorno.pdf

Oviedo, L. (2010). Tercer informe estado de la educación, informe final. Los programas de equidad en la educación costarricense. Recuperado de http://www.estadonacion.or.cr/files/biblioteca_virtual/educacion/003/ Oviedo_2010_programas_equidad.pdf

Pérez, D. (22 julio de 2014). Crece mercado nacional de productos orgánicos. Periódico La Prensa Libre. Recuperado de http://www.anteriores.prensalibre.cr/la_economia.html?start $=185$ 